\section{Inferior Mesenteric Vein Thrombosis}

Liao $\mathrm{PH}$, Wang $\mathrm{CJ}^{*}$ and $\mathrm{Yeh} \mathbf{C H}$

Department of Emergency Medicine, Tungs Taichung Metro Harbor Hospital, Taiwan

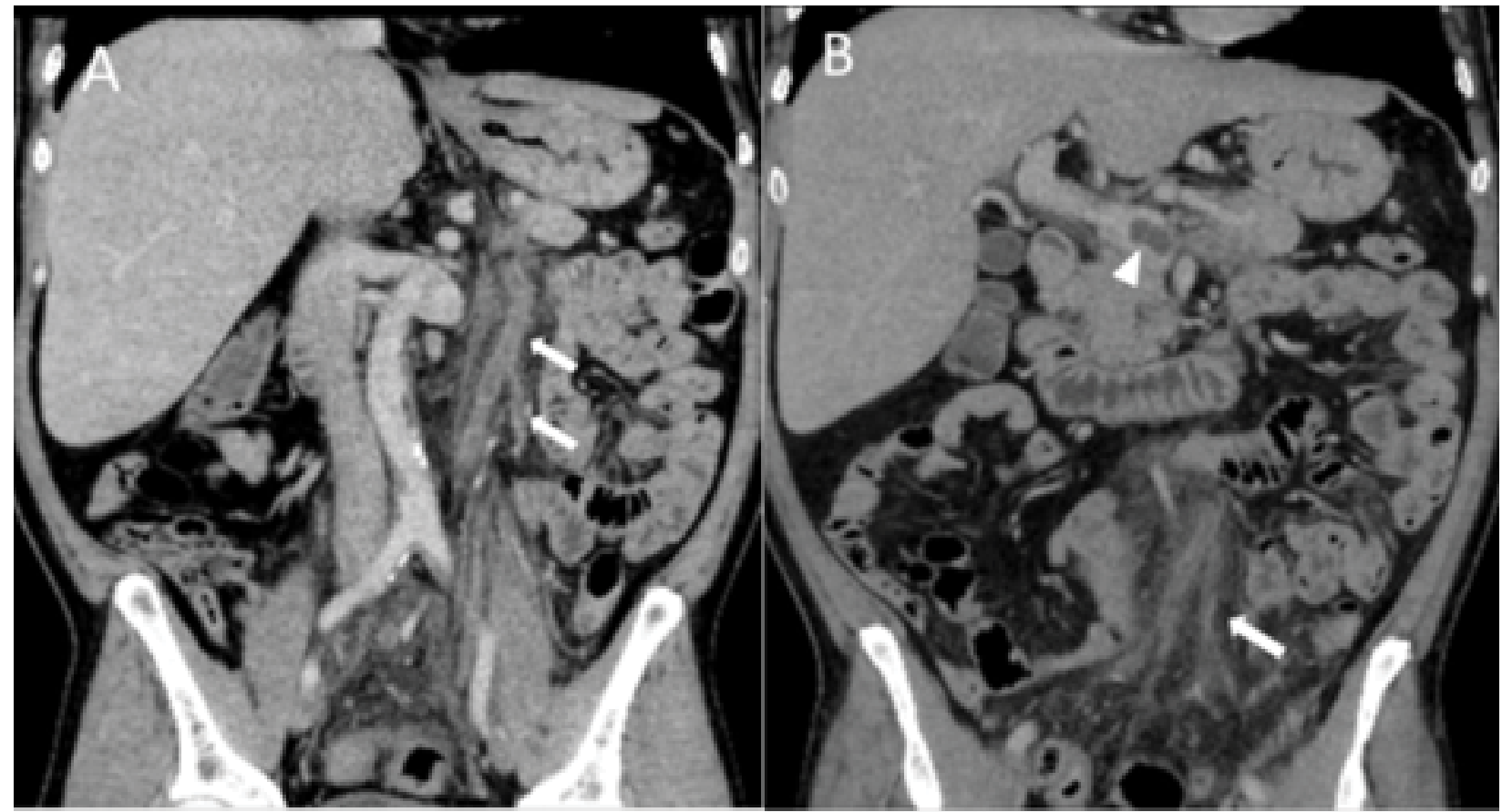

Figure 1: A) Computed tomography scan with contrast demonstrated non-enhanced hypodense inferior mesenteric vein thrombosis and adjacent mesenteric stranding (arrows) B) The non-enhanced hypodense thrombus in the main portal vein (arrowhead), with inflammatory changes around perirectal site with fat standing (arrow).

\section{Clinical Medical Image}

A 50-year-old man with medical history of psychogenic disorder presented to the emergency department with lower abdomen and fullness sensation for 1 week. There were no fever or chills and the vital sign was stable. Physical examination revealed lower abdominal tenderness with rebounding pain. Laboratory reveals WBC 1,7400/ul, GOT/GPT 30/26 CRP 26 mg/dl, BUN/ Cr 6/0.5,D-Dimer $3600 \mathrm{mg} / \mathrm{ml}$. Computed tomography scan with contrast demonstrated non-enhanced hypo dense inferior mesenteric vein thrombosis and adjacent mesenteric stranding (Figure 1A, arrows). In addition, some non-enhanced hypo dense thrombus in the main portal vein (Figure 1B, arrow head) noted. Inflammatory changes around peri-rectal site with fat standing (arrow), which indicated early inferior mesenteric venous thrombosis partial occlusion. There was no evidence of bowel obstruction or ischemia seen on imaging feature, the patient received antibiotic ceftriaxone treatment for 5 days and started with heparin therapy then bridged to warfarin. The comprehensive thrombophilia workup was unremarkable and the blood-culture revealed no organisms. The symptoms resolved without other complication and no signs of recurrent disease were observed 2 month later. Mesenteric ischemia refers to the sudden onset of intestinal hypo perfusion, which usually seen in the setting of thrombophilia or local inflammation. However, the inferior mesenteric venous thrombosis is rare and less common involved. Early diagnosis and management of mesenteric thrombosis can prevent further infarction or surgical intervention [1].

Reference

1. Al Thani H, El Mabrok J, El Menyar A, Al Sulaiti M, Latifi R, et al. (2015) Clinical presentation and outcome of mesenteric vein thrombosis: A single-center experience Angiology 66: 249-256.

*Corresponding author: Che-Jen Wang, Department of Emergency Medicine, Tungs Taichung Metro Harbor Hospital, 699, Sec.8, Taiwan Boulevard, Wuchi, Taichung, 43503,Taiwan,Tel: +886-4-26581919;E-mail: alecstar@mail2000.com.tw

Citation: Liao PH, Wang CJ, Yeh CH (2017) Inferior Mesenteric Vein Thrombosis. Int J Clin Med Imaging 4: 563. doi:10.4172/2376-0249.1000563 